Maria das Graças de Almeida Felipe. "ETHANOL PRODUCTION: ASPECTS TO BE CONSIDERED",

p.547-552. In Luis Augusto Barbosa Cortez (Coord.). Sugarcane bioethanol - R\&D for Productivity and

Sustainability, São Paulo: Editora Edgard Blücher, 2014.

http://dx.doi.org/10.5151/BlucherOA-Sugarcane-SUGARCANEBIOETHANOL_49

\title{
ETHANOL PRODUCTION: \\ ASPECTS TO BE CONSIDERED
}

\author{
Maria das Graças de Almeida Felipe
}

Besides the use of ethanol in flex fuel engines, fuel ethanol has also been used in Brazil as an additive to gasoline (20\% to $25 \%$ ), and also exported to other countries with the objective to reduce GHG emissions.

The impending shortage of oil, the main global energy source, along with the legitimate concerns of society with environmental degradation has created space for a return of fuel ethanol. Produced from biomass, a renewable raw material, this biofuel is now economically attractive. It cab be observed that the transportation sector is one of those with the fastest growth in the world energy matrix, and thus it is not difficult to realize the impact on the world economy. Besides, its use as a fuel blend, alcohol has increasingly been used also as an additive to gasoline to reduce emissions from fossil fuel. Brazil is, at the same time, the largest exporter of ethanol in the world.

These were some of the reasons that led to the creation of the Project on Guidelines for Public Policy for Sugarcane Ethanol in the State of São Paulo, which started in 2006. This project built upon the completion of a series of "workshops" in which subjects that sought to address the entire ethanol fuel production chain were discussed. The main points discussed in the initial event in November 2006 entitled "Ethanol Production" are the subject of this article.

The fermentation process used in Brazil meets several important requirements for the production of ethanol fuel in large scale. Although alcoholic fermentation in its current state meets the needs of ethanol production, there is still ample scope for optimization of current fermentation processes. Within this context the above event brought about a first discussion of key themes: Quality of Raw Materials, Microbial Physiology and Ecology, Fermentation and Distillation processes. The main objective was to stimulate discussion of ways to improve the fermentation process, and to find solutions and reduce its cost further, to meet the large expansion of ethanol production and transforming the sugar mills and distilleries into large producers of biofuels.

The discussions resulting from this first event are presented here in this chapter, translated into topics, by questions and provocations posed by the workshop.

\section{QUALITY OF THE RAW MATERIAL}

The purpose of this subject is to discuss the prerequisites that must meet the raw materials involved in fermentation to ensure efficiency in the current conditions and projected scenarios. The quality of sugarcane (basic raw material) should be considered as the main factor to be taken into account to improve the performance of the alcoholic fermentation. Currently commercial production of ethanol only one third of the biomass is utilized, that is the sugar extracted from the stems in the broth (sucrose). Thus, the need for assessing parameters such as harvesting, loading and transportation of sugarcane due to the impacts exerted on the production process. These operations are not only expensive, but result in loss of 
raw materials and contribute to worsen the quality of this material.

Collecting also the cane tops during the harvest may result in problems in the industry as impurities (soil and mineral) which will require advances in mechanization. Determining where losses do occur poses the question whether current knowledge is sufficient to reduce these losses. So it becomes urgent to review the methods for determining quality (impurities of raw materials), and how best to implement them. This would avoid problems in the industry due to the importance of knowing, as soon as possible, the quality of raw material entering the process.

Studies on the correlation between physiological condition of the plant and industry are needed, if improvements of the production process are to be achieved. This could be related to raw material derived from the broth and therefore would be possible to know, for example, if the sterilization before fermentation, via radiation, could allow the use of specific-yeasts rather than the "rustic" yeasts currently used. Moreover, another possibility would be to clarify instead of obtaining the vinasse. There is, however, some concern because this procedure could cause problems for the yeasts. There is also a problem with the current practice of using insecticides to control pests and thus it is very important to know how to minimize their use so not to compromise the fermentation.

\section{PHYSIOLOGY AND MICROBIAL ECOLOGY}

Our understanding of the physiological conditions imposed by industrial microorganisms processes is undoubtedly very important so that we can identify the physical, chemical and microbiological contaminants that contribute to exercise stress effects or stimulants on the agents of fermentation (yeast) or contaminants (bacteria and other yeasts).

In commercial ethanol production several factors are already known that contribute to reducing the fermentative efficiency e.g. bacterial contamination, high foaming, high residual sugar, yeast flocculation, overgrowth or reduced growth.
We can add to these the formation of unwanted by-products to the process such as glycerol and organic acids (acetate, succinate, malate, lactate). In this context, the critical point is presented as a certainty that the organism has a priority of its metabolism to generate energy - ATP for the formation of new cells. It is clear then that the need is not essential microbial conversion of sugars in the product in our concern as ethanol or of products formed by cellular stress such as glycerol and organic acids. It is also clear that the priority of the yeast is to maintain their survival, incompatible with our need. This leads to the need to direct the conditions of the fermentation process so that the metabolic flux is directed primarily to the formation of the target product, that is, ethanol.

In this sense it is important to understand what and how certain parameters contribute to the formation of these products and how they could the microbial metabolism be affected to favor the bioprocess. This would allow the maximum conversion of carbon source in ethanol at the same time that the diversion to other compounds (products) to be minimized. Another aspect of the approach is the physiological conditions of yeast in the fermentation carried out with cell recycle and also those with the use of immobilized cells, combining the techniques of molecular biology which could help to establish a sustainable fuel ethanol industry in the light of new changes that might be imposed on the production system.

Evaluating the influence factors prior to fermentation and the presence of metals in soil mixed with sugarcane was also highlighted. Metals such as iron may be responsible for physiological and biochemical changes during fermentation due to adsorption on the wall of the yeast cell flocculation and consequently after the centrifugation step.

As a recommendation to increase ethanol productivity, it was clear that the methodology should involve industrial production sectors of the yeast physiological approaches, identification and control of the stressors imposed by the industrial process in "real time". Research targets were also suggested e.g. changing the stoichiometry of fermentation to increase the formation of ethanol, obtaining alternative ways to control bacterial 
contamination and the use of bacteriocins as well as yeast strains suitable for industrial processes.

\section{FERMENTATION}

The fermentation process used in Brazil meet several important requirements for ethanol production on a large scale; Brazil is the most advanced country in terms of technology in the production of this biofuel. However, despite the current stage of development is still possible to improve its performance. Measures to improve fermentation include production of sugarcane varieties with higher content of fermentable sugars, increased ethanol fermentation efficiency to values higher than today (0.511 grams of ethanol per gram of reducing sugar as glucose). Other factors that interfere in this process step are reduction fermentation temperature, consumption of sulfuric acid or even its replacement, the cost of centrifugation and cell recycling, and in the volume of stillage generated. Although the increase in alcohol content (average value is around 8.5\% (vol.), the use of alternatives to use antibiotics, nitrogen and potassium, the standardization of methods of analysis of fermentation, merit new proposals for other alternative to current processes.

The main approach during the discussion on fermentation was that the present model of ethanol production in Brazil uses a route-winning technology that makes it difficult to compete with other alternatives. This is a mature process (raw material represents $60 \%$ of the cost) and only small gains can be achieved that require large investment. Other proposed routes are certainly fertile ground for research and technological development; instruments to stimulate innovation in this area were used for long term funding or venture partnerships remain small.

For the vast expansion expected within the next 20 years, it will be necessary to further optimize production to lower cost; this requires considerable research effort to make the current process more reliable and productive, and adapted to the new conditions particularly in less favorable planting areas.
Highlighted was also the fact that only part of the cane is utilized, as burning still occurs eliminating almost two thirds of the available energy, while the actual content of soluble sugar arriving at the processing is low. This points out to significant gains may occur by optimizing these aspects, although very little has actually been done in this area. Also, the fact that the current processing is based on abundant availability of energy (in the form of bagasse, which had no other use) generates a huge waste for energy e.g. to extract the juice though the control loss is quite efficient. Exception is the fermentation process that can be achieved $90 \%$ of its maximum efficiency (0.511 kilograms ethanol per kilogram of sugar). Ethanol, although contains a considerable amount of usable energy from sugar, is also a huge waste of materials (more than $50 \%$ of the sugar does not enter as final products).

In addition to the limit of the maximum output of the fermentation process we have to consider that this is an integrated industrial process, both in terms of use the raw material (sugar) for energy generation required for processing, and also the co-production of sugar. This strategy of diversification and integration beyond the fact that sugarcane can not be stored, and that its composition evolves during the collection period, leads to the necessity that the process must have other characteristics e.g. high flexibility due to variations such as the quantity and quality of raw materials and high resilience (ability to recover after the changes); although it is questionable whether any surviving populations are adequate to obtain a low cost alcohol. In this context further research is needed is needed on storage, extending sugarcane harvest; and whole sugarcane harvesting.

The fermentation process has a good productivity, efficiency without sterilization (compared to other industrial fermentation processes), although these factors could theoretically at least be improved with better understanding of the physiology of populations of yeast in industrial environment (mainly subjected to many stressful conditions such as recycling and treatment acid), with better knowledge of the stoichiometry (metabolic pathways) of biodiversity. It is necessary to 
develop new alternatives more viable and sustainable than the current microbiological control and more strains of yeast for the start of fermentation process.

The kinetics of the fermentative process needs to be well understood to relate it better to the best components of the environment affected by the composition of the raw material. Also, the fact that the final content of ethanol is still too low in the broth, leads to overloading of the separation process while fermentation temperature must be kept low in view of the toxicity of ethanol and the presence of thermophilic bacteria.

More direct and simpler alcohol production processes using whole sugarcane is needed; more like a chemical synthesis to take advantages of both routes. Significant reductions in the cost of production have been achieved over the past 30 years shows the need for research either in their own production units or conditions (environment) with real limitations and particularities. To do so will require much more funding and research.

\section{DISTILLATION}

Considerable changes have taken place in Brazil ethanol distillation, with significant increases in productivity devices such as reducing the consumption of steam per liter of ethanol for both the hydrous and anhydrous. The main goal of distilling alcohol is to get a wine with high levels of alcohol corresponding to values from $92.6 \%$ to $93.5 \%$ and greater than $99.3 \%$ by weight ( ${ }^{\circ} \mathrm{INPM}$ ) for the hydrated and anhydrous respectively. The wine also contains small amounts of contaminants from the fermentation process itself and other alcohols, aldehydes, organic acids and others. The removal of these compounds, mainly of higher alcohols, is required as their presence affect the quality of ethanol. Within this context, the discussion was based on several aspects such as the reductions in energy consumption in distillation and losses in the process, the introduction of processes of multiple effects with a consequent reduction in energy consumption and cooling water, re-evaluating apparatus and processes used for both hydrous and anhydrous ethanol in order to increase production; the recovery of diluted products and also the introduction of sensors and automation.

Research proposals relevant to ethanol distillation in the near future from the workshop are listed below:

- feasibility of concentrated broth as well as higher alcohol content in wine that could be achieved by $16{ }^{\circ} \mathrm{GL}$ to allow the reduction in steam consumption;

- increasing the number of trays in columns and its effect on energy consumption;

- changes in construction and/or operating columns associated with the extraction of products to expand the main product (hydrated ethanol);

- development for process control systems, and energy efficient generation of products and by-products of better quality;

- better economic use of byproducts such as superior alcohols;

- development of basic configurations columns for producing hydrated ethanol fuel; the more flexible distillation units to allow the production of alcohols of different quality standards;

- investigate double integration type thermal effect for distilling alcoholic considering the difficulties as risks of formation of incrustations and problems with flow velocities in the trays;

- utilization of integrations in the process of thermal dehydration linking alcohol use to the quality and use of anhydrous alcohol, including requirements of foreign markets;

- development of new bins and fillings for distillation columns and their impact on energy consumption and investment costs;

- development of new materials for the process enabling plants pervaporation and scale appropriate to the national market or new type zeolite materials for domestic production;

- application of current knowledge in other industrial processes such as petrochemical industry with emphasis on tools for advanced process control. 
It is also important to consider that the solutions to the problem of distillation are not restricted to the distillery itself due to interference exerted by the quality of the raw material which influences the cost and efficiency of its subsequent processing. Multiple effect distillation to reduce steam consumption in the distillation can be compromised due to the risk of fouling which is also a consequence of the quality of the raw material for fermentation, or the wine.

\section{FINAL CONSIDERATIONS}

The main conclusions/considerations that may be highlighted from the Fapesp workshop ${ }^{1}$ are:

Future demand for sustainable renewable energy is signalized with a set of challenges that require specific actions, coordinated with incentives and subsidies that can turn them into viable resources internationally, including biofuels such as sugarcane ethanol. To increase their participation in the global energy matrix, needs to carry out research in several areas that has a direct impact in the production process e.g. the quality of raw materials, physiology and microbial ecology, fermentation and distillation.

There was consensus among many participating researchers in the workshop for a coordinated effort across the whole ethanol supply chain, rang-

\footnotetext{
1 The information presented in this chapter originated from the Workshop Ethanol Production, the first of a serie of events of the Fapesp PPP Ethanol Project Diretrizes de Políticas Públicas para a Agroindústria Canavieira do Estado de São Paulo, occurred at the Escola de Engenharia de Lorena/USP November $10^{\text {th }}, 2006$. The main speakers, and collaborators are listed below:

Quality of raw material: Dr. Carlos Eduardo Vaz Rossell - CTBE (speaker/reporter); Dr. Márcia Justino R. Mutton - Unesp and Dr. Jorge Horii - Esalq/USP (debaters). Physiology and Microbial Ecology: Dr. Luiz Carlos Basso - Esalq-USP (speaker/reporter); Dr. Márcia Justino R. Mutton - Unesp and Dr. Maria das Graças de Almeida Felipe - EEL/USP (debaters).

Fermentation: Eng. Jaime Finguerut - CTC (speaker/ reporter); Dr. Henrique Vianna Amorim - Fermentec and Dr. Francisco Maugeri Filho - Unicamp (debaters).

Distillation: Dr. Antonio José de A. Meirelles - Unicamp (speaker/reporter); Dr. Rubens Maciel Filho - Unicamp and MSc. Jonas Nolasco - Dedini (debaters).
}

ing from agricultural to industrial pahses. Without this coordinated effort will be difficult to achieve the degree of competitiveness necessary to increase production of ethanol in the next 20 years.

The quality of raw material is clearly a major impact on the production process which requires the evaluation of parameters such as harvesting, transport and storage, to improve the performance of the alcoholic fermentation. Therefore, it is imperative that we take a set of actions and a review of methods for determining the quality, and at the same time to ensure they are more precise and faster implemented. This includes studies on the physiology of the plant in order to evaluate its correlation with the industrial problems.

With regard to physiology and microbial ecology, it is essential to understand how the industrial process interferes with the microbial metabolism so that it can identify the physical, chemical or microbiological benefit or those that harm the process. Increasing knowledge on the microbial metabolism in relation to fermentation is necessary so that we can achieve maximum process efficiency and reduce the amount of unwanted byproducts. To achieve this it is necessary to direct the process conditions in order to prioritize the metabolic flux of targeted product (e.g. ethanol) rather than the microbial needs is the formation of energy in the form of ATP.

It can be concluded that the efficiency of the fermentation can be increased, despite the advanced level of technology today. This requires knowledge of factors such as the physiology of yeasts in industrial environments, the availability of new more viable sustainable alternatives, current microbiological control and the development of new strains of yeast. Also the evaluation of interfering factors such as the temperature decreases, the cost of centrifugation and cell recycling as well as the volume of vinasse will help improve the process. At the same time it is important to have a better understanding of the kinetics of the fermentation process so that they can properly assess the effects that the composition of the raw material can cause to this process. It is also necessary to standardize methods of analysis and evaluation of other alternatives to current processes. It is 
important that research be done as far as possible in production units or close to them.

Similarly, the fermentation stage of the wine distillation also has made significant advances to reach the current stage. However, the implementation of some measures may allow to improve this process. Among these could be mentioned the use of concentrated grape and higher alcohol content in wine, upgrading of equipment and processes in place for both hydrous and anhydrous; best economic use of byproducts such as superior alcohols as well as improving the quality of and products generated; the introduction of sensors and auto- mation, and the application of current know-how in other process areas such as petrochemicals. It should also be considered that, similar to what occurs in the fermentation process, the distillation process also affects the quality of raw material thereby requiring better control to avoid losses.

As a result of this meeting of experts, it can be said that the contribution to the development of the sugar-ethanol sector with respect to the first generation ethanol, could offer in addition to improving production efficiency, the introduction of new innovative processes, cost-effective and with minimum environmental impacts. 